\title{
Aggregative Games and Best-Reply Potentials
}

\author{
Martin Kaae Jensen
}

the date of receipt and acceptance should be inserted later

\begin{abstract}
This paper introduces quasi-aggregative games and establishes conditions under which such games admit a best-reply potential. This implies existence of a pure strategy Nash equilibrium without any convexity or quasi-concavity assumptions. It also implies convergence of best-reply dynamics under some additional assumptions. Most of the existing literature's aggregation concepts are special cases of quasi-aggregative games, and many new situations are allowed for. An example is payoff functions that depend on own strategies as well as a linear combination of the mean and the variance of players' strategies.
\end{abstract}

Keywords Aggregative Games, Aggregation, Strategic Substitutes, Nash Equilibrium, Potential Game, Best-reply Dynamics.

JEL-classification C72.

I would like to thank Daron Acemoglu, Burkhard Schipper, and an anonymous referee for their helpful remarks and comments. Thanks also to seminar participants at the University of Copenhagen and University of Warwick. All remaining errors are of course my responsibility.

Martin Kaae Jensen

Department of Economics, University of Birmingham, 90 Vincent Drive, Birmingham B15 2TT, UK. Email: m.k.jensen@bham.ac.uk Homepage: http://socscistaff.bham.ac.uk/jensen/ 


\section{Introduction}

In the standard formulation of a non-cooperative game, a player's payoff function depends on opponents' individual strategies. Yet, in many games, payoff functions depend only on some aggregate of these, an example being the Cournot model where it is the aggregate supply of opponents that matters rather than their individual strategies. Within recent years such aggregative games have attracted considerable attention, leading to papers on evolutionary stability, Knightian uncertainty, learning dynamics, and evolution of preferences, to mention a few. ${ }^{1}$

This paper's two main objectives are, firstly, to define a new and very general notion of aggregation, called quasi-aggregation, and secondly, to prove that if all best-reply selections are either increasing or decreasing, quasi-aggregative games are best-reply potential games (Voorneveld (2000)). If it is only assumed that some selection has this property, the games are pseudo-potential games (Dubey et al. (2006)). A direct consequence of these results is that such games have a pure strategy Nash equilibrium (PSNE) irrespective of whether strategy sets are convex or payoff functions quasi-concave. A second consequence is that Liapunov methods can be used to address their stability properties. Thus it is shown, for example, that with singlevalued best-replies, a unique PSNE will always be globally stable under the game's myopic best-reply dynamics. If best-replies are not single-valued, the same is true provided that certain "perverse" paths are ruled out.

The results can be seen as a direct continuation of Dubey et al. (2006), who define pseudo-potential games and show that any game with linear aggregation and an increasing or decreasing continuous best-reply selection, belongs to this class. The authors also extend this observation to aggregation rules which are only required to be symmetric and linear in any single player's strategy. ${ }^{2}$ The cases studied by Dubey et al. (2006) and generalized in Kukushkin (2005) are special cases of this paper's class of games. Hence they lead to best-reply potential games if all best-replies are decreasing and pseudo-potential games if some selection is decreasing (whether or not this selection is continuous). That a game is a best-reply potential game and not some weaker notion of a potential game, plays a crucial role for our main stability results. It is obviously also crucial if, say, one wants to apply the robustness results of Morris and Ui (2004) (which, to be sure, concerns best-reply potential games).

Quasi-aggregative games also include as a special case Alos-Ferrer and Ania (2005)'s notion of a generalized symmetric aggregative game (see also Schipper (2005)). In particular it will follow that a PSNE exists in such games under AlosFerrer and Ania's assumptions. Generalized symmetric aggregation is in some respects the aggregator concept which is most closely related to this paper's. Neither

\footnotetext{
1 See e.g. Morris and Ui (2004), Possajennikov (2003), Alos-Ferrer and Ania (2005), Schipper (2005), and Kockesen et al. (2000). For a long list of specific examples see Alos-Ferrer and Ania (2005). The concept of aggregation in that paper ("generalized symmetric aggregation") is returned to below.

2 The mentioned class will be considered in section 2.3.3 of this paper. See also Kukushkin (2005), who proves that such games admit a so-called Cournot potential if all best-reply selections are either increasing or decreasing. A Cournot potential is, roughly, an order theoretic equivalent to a generalized best-reply potential.
} 
assumes that aggregators have a specific functional form, and in both cases, the permissible functions can be viewed as the general solution to a set of functional equations. ${ }^{3} \mathrm{~A}$ difference is that quasi-aggregators are not required to be symmetric functions. A somewhat more fundamental difference is that in quasi-aggregative games, strategy sets may be multidimensional (see the first application in section 5 for an example with multi-dimensional strategy sets).

The organization of this paper is as follows: Section 2 contains the basic definitions, assumptions, and describes various special cases of quasi-aggregative games (existing as well as new ones). Sections 3-4 contain the main results (the proofs are relegated to section 6 at the end of the paper). Finally, section 5 contains two applications to games which are not covered by any of the previously mentioned aggregation concepts.

\section{Definitions and Assumptions}

Let $\Gamma=\left(\tilde{\pi}_{i}, S_{i}\right)_{i \in \mathscr{I}}$ denote a non-cooperative, pure strategy game with a finite set of players $\mathscr{I}=\{1, \ldots, I\}$, and finite dimensional strategy sets $S_{i} \subseteq \mathbb{R}^{N}$. The joint strategy set $S=\prod_{i \in \mathscr{I}} S_{i}$, is assumed to be a compact metric space, and payoff functions $\tilde{\pi}_{i}: S \rightarrow \mathbb{R}, i \in \mathscr{I}$ are assumed to be upper semi-continuous. The best-reply correspondences $\tilde{R}_{i}\left(s_{-i}\right)=\arg \max \left\{\tilde{\pi}_{i}\left(s_{i}, s_{-i}\right): s_{i} \in S_{i}\right\}, s_{-i} \in S_{-i}=\prod_{j \neq i} S_{j}, i \in \mathscr{I}$, are consequently non-empty valued and upper hemi-continuous. No other standard assumptions such as quasi-concavity of payoff functions and convexity of strategy sets, are imposed.

\subsection{Quasi-Aggregative Games}

Consider a game such as the Cournot model, $\tilde{\pi}_{i}(s)=s_{i} P\left(s_{i}+\sum_{j \neq i} s_{j}\right)-C_{i}\left(s_{i}\right)$ (here $P$ is the inverse demand function and $C_{i}$ the cost function of firm $\left.i\right)$. Defining $\sigma_{i}\left(s_{-i}\right)=$ $\sum_{j \neq i} s_{j}$ we can write: $\tilde{\pi}_{i}(s)=\pi_{i}\left(\sigma_{i}\left(s_{-i}\right), s_{i}\right), i \in \mathscr{I}$. Intuitively, players only influence each other through the terms $\sigma_{i}\left(s_{-i}\right)=\sum_{j \neq i} s_{j}$, called the interaction functions. Defining $g(s)=\sum_{i} s_{i}$, it is furthermore true that $g(s)=F_{i}\left(\sigma_{i}\left(s_{-i}\right), s_{i}\right)$ for all $i$ where $F_{i}\left(\sigma_{i}\left(s_{-i}\right), s_{i}\right)=\sigma_{i}\left(s_{-i}\right)+s_{i}$. In words, there exists a function $g$, the aggregator, the value of which $g(s)$ can always be calculated (via $F_{i}$ ) from knowledge of a player $i$ 's strategy and the interaction term $\sigma_{i}\left(s_{-i}\right)$. These two abstract features of the Cournot model form the basis of this paper's central definition:

Definition 1 (Quasi-Aggregative Games) The game $\Gamma=\left(\tilde{\pi}_{i}, S_{i}\right)_{i \in \mathscr{I}}$ is said to be $a$ quasi-aggregative game with aggregator $g: S \rightarrow \mathbb{R}$, if there exist continuous functions $F_{i}: \mathbb{R} \times S_{i} \rightarrow \mathbb{R}$ (the shift functions), and $\sigma_{i}: S_{-i} \rightarrow X_{-i} \subset \mathbb{R}, i \in \mathscr{I}$ (the interaction functions) such that each of the payoff functions $i \in \mathscr{I}$ can be written:

$$
\tilde{\pi}_{i}(s)=\pi_{i}\left(\sigma_{i}\left(s_{-i}\right), s_{i}\right)
$$

\footnotetext{
${ }^{3}$ For the concept of a functional equation, see Aczél (2006).
} 
where $\pi_{i}: X_{-i} \times S_{i} \rightarrow \mathbb{R}$, and:

$$
g(s)=F_{i}\left(\sigma_{i}\left(s_{-i}\right), s_{i}\right), \text { for all } s \in S \text { and all } i \in \mathscr{I}
$$

Remark 21 As is clear from the definition, a quasi-aggregative game is an ordinal concept, i.e., all functions involved are only determined up to a strictly monotonic transformation (which may be either strictly increasing or strictly decreasing). ${ }^{4}$

An alternative, but less general way of defining a quasi-aggregative game replaces (1) in the definition with:

$$
\tilde{\pi}_{i}(s)=\hat{\pi}_{i}\left(g(s), s_{i}\right)
$$

Here $\hat{\pi}_{i}: X \times S_{i} \rightarrow \mathbb{R}$ and $X=g(S) \subseteq \mathbb{R}$. In this formulation, payoff functions can be written as a function of $s_{i}$ and the aggregate $g(s)$. Since one may simply insert (2) into (3) in order to get (1): $\pi_{i}\left(\sigma_{i}\left(s_{-i}\right), s_{i}\right)=\hat{\pi}_{i}\left(F_{i}\left(\sigma_{i}\left(s_{-i}\right), s_{i}\right), s_{i}\right)$, it is clear that (3) is a special case of (1) when $g$ can be made to satisfy the conditions in (2) for suitably chosen functions $F_{i}$ and $\sigma_{i}, i \in \mathscr{I}$. These shift and interaction functions will then play an auxiliary role, although the latter will always have the aforementioned game theoretic interpretation as "the channels through which players exert influence on each other". Thus in the Cournot model, $\tilde{\pi}_{i}(s)=\hat{\pi}_{i}\left(g(s), s_{i}\right)$ where $g(s)=\sum_{i} s_{i}$ and $\hat{\pi}_{i}\left(g(s), s_{i}\right)=s_{i} P(g(s))-C_{i}\left(s_{i}\right)$. Associated shift and interaction functions were found at the beginning of this section. Notice how the argument now begins with knowledge of the aggregator $g$ and proceeds to determine the functions $F_{i}$ and $\sigma_{i}$, $i \in \mathscr{I}$.

The main feature of a quasi-aggregative game is captured by the weak separability conditions in (1). As in the Cournot example above, players only influence each other through certain real-valued functions, $\sigma_{i}: S_{-i} \rightarrow \mathbb{R}$. These functions are not assumed to be linear, however. They are now allowed to take any form compatible with the existence of an aggregator $g$ and shift functions $F_{i}, i \in \mathscr{I}$ such that (2) holds. (2) is a system of functional equations and any solution, or class of solutions, will form a special case of quasi-aggregative games. In section 2.3 a number of such solutions will be considered and as will become clear the class of permissible functional forms is substantial. There is one set of solutions which is well known and worth mentioning already here, however. (2) will be satisfied for strictly monotonic functions $F_{i}$ and $\sigma_{i}$ provided that $g$ is additively separable (Gorman (1968)). The converse is true as well: if (2) holds for strictly monotonic functions $F_{i}$ and $\sigma_{i}$, then $g$ will be additively separable. If one's starting point is payoff functions in the form (3) where $g$ is additively separable, the game is consequently quasi-aggregative (see section 2.3.2 for further details). It is important to stress, then, that this paper does not assume that $F_{i}$ and $\sigma_{i}$ are monotonic, in particular $g$ is not (implicitly) assumed to be additively separable. In fact, this paper's only assumption in this respect (assumption 2) will be seen to be a second order condition. As such no monotonicity assumptions are placed on any function what so ever. ${ }^{5}$ Nonetheless, nothing prevents us from thinking of (2)

\footnotetext{
4 Hence the term quasi-aggregative. The observation is trivial for the interaction functions. It is also clear that (2) will hold for functions $g$ and $F_{i}, i \in \mathscr{I}$ if and only if it holds for $\tilde{g}=h \circ g$ and $\tilde{F}_{i}=h \circ F_{i}$, $i \in \mathscr{I}$ where $h: \mathbb{R} \rightarrow \mathbb{R}$ is either strictly increasing or strictly decreasing.

5 The simplest example of a function admitted by (2) which is not additively separable is $g(s)=\prod_{i} s_{i}$ when $0 \in S_{i}$ for some $i$.
} 
as weak "independence" conditions (cf. Gorman (1968), Vind and Grodal (2003)) which, crucially, are imposed without any associated monotonicity or sensitivity assumptions.

Before turning to assumptions and examples, let us introduce a direct generalization of quasi-aggregative games. All of this paper's results remain valid for this larger class of games. In sections 2.3.3-2.3.5 we shall encounter games which are generalized quasi-aggregative but (typically) not quasi-aggregative.

Definition 2 (Generalized Quasi-Aggregative Games) The game $\Gamma=\left(\tilde{\pi}_{i}, S_{i}\right)_{i \in \mathscr{I}}$ is said to be generalized quasi-aggregative if it satisfies definition 1 with (2) replaced by the weaker set of conditions:

$$
g(s)=F_{i}\left(\sigma_{i}\left(s_{-i}\right), s_{i}\right)+v_{i}\left(s_{-i}\right), \text { for all } s \in S \text { and all } i \in \mathscr{I}
$$

where $v_{1}, \ldots, v_{I}$ may be arbitrary real-valued functions.

As can be seen, the only difference between this definition and definition 1 , is that the set of permissible functional forms has been expanded. On the downside, a generalized quasi-aggregative game is not subject to arbitrary monotonic transformations of the aggregation conditions (4). Indeed, any monotonic transformation $h$ of $g$ and the $F_{i}$ 's must be affine, $h(z)=a z+b$ where $a \in \mathbb{R} \backslash\{0\}, b \in \mathbb{R}$. A generalized quasiaggregative game is therefore, in some sense, an aesthetically less pleasing concept than a quasi-aggregative game.

We end this section with an example of a function $g$ that does not satisfy (4) for any choice of functions $F_{i}, \sigma_{i}$, and $v_{i}, i \in \mathscr{I}$. It follows that there are games of the form (3) that cannot be (generalized) quasi-aggregative.

Example 1 Consider a game with three players whose payoff functions satisfy (3) for $g\left(s_{1}, s_{2}, s_{3}\right)=\frac{1}{2} s_{1}^{2} s_{2}+\frac{1}{3} s_{1}^{3} s_{3}+s_{1} s_{2}$ (here $S_{i} \subseteq \mathbb{R}, i=1,2,3$, and it is assumed that $S_{2}$ contains at least two distinct strategies). There do not exist functions $\sigma_{i}, F_{i}$, and $v_{i}$, $i=1,2,3$, such that (4) holds. If (4) were to hold for player $i=1$, it would follow that (*) $D_{s_{1}} g(s)=D_{s_{1}} F_{1}\left(\sigma_{1}\left(s_{-1}\right), s_{1}\right)$. (*) implies that for any fixed $\hat{s}_{1} \in S_{1}$, the derivative $D_{s_{1}} g\left(\hat{s}_{1}, \cdot, \cdot\right)$ is constant on any of the sets $\Delta_{1}\left(\alpha, s_{1}\right)=\left\{\left(s_{2}, s_{3}\right) \in S_{2} \times S_{3}: D_{s_{1}} g(s)=\right.$ $\alpha\}=\left\{\left(s_{2}, s_{3}\right): s_{1} s_{2}+s_{1}^{2} s_{3}+s_{2}=\alpha\right\}, s_{1} \in S_{1}$ and $\alpha \in g(S)$. But this is not true. For example consider $D_{s_{1}} g\left(2, s_{2}, s_{3}\right)=2 s_{2}+4 s_{3}+s_{2}$ on the set $\Delta_{1}(1,1)=\left\{\left(s_{2}, s_{3}\right)\right.$ : $\left.s_{3}=1-2 s_{2}\right\} . D_{s_{1}} g\left(2, s_{2}, s_{3}\right)$ will be constant only if $2 s_{2}+4-8 s_{2}+s_{2}=4-5 s_{2}$ is constant and this is not the case.

\subsection{Assumptions}

We shall be needing two assumptions throughout. Recall from definition 1 that $\sigma_{i}$ : $S_{-i} \rightarrow X_{-i} \subseteq \mathbb{R}$. In the following it is convenient to set $X_{-i}=\sigma_{i}\left(S_{-i}\right)$ (the range of $\left.\sigma_{i}\right)$. When a game is (generalized) quasi-aggregative, it will be true that $\tilde{R}_{i}\left(s_{-i}\right)=$ $R_{i}\left(\sigma_{i}\left(s_{-i}\right)\right)$ where $R_{i}: X_{-i} \rightarrow 2^{S_{i}}$ is given by $R_{i}\left(x_{-i}\right)=\arg \max \left\{\pi_{i}\left(x_{-i}, s_{i}\right): s_{i} \in S_{i}\right\}$. Intuitively, $R_{i}$ is the "reduced" best-reply correspondence which describes how agent $i$ 's best-replies depend on the interaction term $x_{-i}=\sigma_{i}\left(s_{-i}\right)$. Our first assumption is placed directly on these correspondences. 
Assumption 1 Each of the correspondences $R_{i}: X_{-i} \rightarrow 2^{S_{i}}$ has a decreasing selection, i.e., there exists a function $r_{i}: X_{-i} \rightarrow S_{i}$ with $r_{i}\left(x_{-i}\right) \in R_{i}\left(x_{-i}\right)$, all $x_{-i} \in X_{-i}$ such that $r_{i}\left(\tilde{x}_{-i}\right) \geq r_{i}\left(x_{-i}\right)$ whenever $\tilde{x}_{-i} \leq x_{-i}$.

Sometimes we shall also consider the following strengthened version of assumption 1:

Assumption 1' Each of the correspondences $R_{i}: X_{-i} \rightarrow 2^{S_{i}}$ is strictly decreasing (i.e., every selection from $R_{i}$ is decreasing).

Remark 22 Assumption 1 will be satisfied under the conditions of Topkis' Theorem (Topkis (1998)): Each $S_{i}$ a lattice, every $\pi_{i}\left(x_{-i}, s_{i}\right)$ supermodular in $s_{i}$, and exhibiting decreasing differences in $x_{-i}$ and $s_{i}$ (for the relevant definitions see Topkis (1998). The definition of decreasing differences plays a separate role in this paper and is reproduced right after this remark). If strategy sets are one-dimensional, the first two of these requirements will automatically be satisfied, so if $\pi_{i}$ is $C^{2}$ an easily checked sufficient condition is that $D_{x_{-i} s_{i}}^{2} \pi_{i}\left(x_{-i}, s_{i}\right) \leq 0$. Assumption $1^{\prime}$ holds in this case if the inequality is strict: $D_{x_{-i} s_{i}}^{2} \pi_{i}\left(x_{-i}, s_{i}\right)<0$.

Recall that $F_{i}: X_{-i} \times S_{i} \rightarrow \mathbb{R}$ exhibits strictly increasing (decreasing) differences if $F_{i}\left(\tilde{x}_{-i}, \tilde{s}_{i}\right)-F_{i}\left(x_{-i}, \tilde{s}_{i}\right)>(<) F_{i}\left(\tilde{x}_{-i}, s_{i}\right)-F_{i}\left(x_{-i}, s_{i}\right)$ whenever $\tilde{x}_{-i}>x_{-i}$ and $\tilde{s}_{i}>s_{i}$ (Topkis (1998)).

Assumption 2 The shift-functions $F_{i}, i \in \mathscr{I}$, all exhibit strictly increasing differences in $x_{-i}$ and $s_{i}$ (possibly after a strictly monotonic transformation).

Because the interaction and shift functions are only determined up to strictly monotonic transformations (affine in the case of $F_{i}$ when the game is generalized aggregative), assumption 1 may be replaced by the assumption that each of the correspondences $R_{i}$ has an increasing selection. This is so because we may write, $R_{i}\left(\sigma_{i}\left(s_{-i}\right)\right)=$ $R_{i}\left(-\tilde{\sigma}_{i}\left(s_{-i}\right)\right)$ where $\tilde{\sigma}_{i}\left(s_{-i}\right)=-\sigma_{i}\left(s_{-i}\right)$, and in terms of definitions 1-2 we then replace $g$ with $\tilde{g}(s)=-g(s)$ and $F_{i}$ with $\tilde{F}_{i}\left(\tilde{\sigma}_{i}\left(s_{-i}\right), s_{i}\right)=-F_{i}\left(-\tilde{\sigma}_{i}\left(s_{-i}\right), s_{i}\right)$. Clearly, $\tilde{F}_{i}\left(x_{-i}, s_{i}\right)$ exhibits strictly increasing differences in $x_{-i}$ and $s_{i}$ if and only if $F_{i}$ does. So assumption 2 will still hold. For much the same reason, assumption 2 can be replaced with the assumption that each $F_{i}$ exhibits strictly decreasing differences (simply take $\tilde{g}(s)=-g(s)$ and $\tilde{F}_{i}\left(x_{-i}, s_{i}\right)=-F_{i}\left(x_{-i}, s_{i}\right)$ so that $\tilde{F}_{i}$ exhibits strictly increasing differences). In fact, any "permutation" is clearly seen to be allowed: For example, we could assume that for all $i$, either (i) $R_{i}$ has an increasing selection and $F_{i}$ exhibits strictly increasing differences, or (ii) $R_{i}$ has a decreasing selection and $F_{i}$ exhibits strictly decreasing differences. This allows for a mixture of strategic substitutes and strategic complements across the agents.

If each interaction function $\sigma_{i}$ is coordinatewise decreasing and assumption 1 holds, then every best-reply correspondence $\tilde{R}_{i}, i \in \mathscr{I}$ will have an increasing selection (namely the composition $r_{i}\left(\sigma_{i}\left(s_{-i}\right)\right) \in \tilde{R}_{i}\left(s_{-i}\right)$, where $r_{i}$ is the selection from assumption 1). Dubey et al. (2006) call a game with an increasing best-reply selection a game of weak strategic complements (WSTC). Similarly, the game will be 
a game of weak strategic substitutes (WSTS) if each $\sigma_{i}$ is coordinatewise increasing and assumption 1 holds. ${ }^{6}$ Assumptions 1-2 do not imply that the interaction functions are either decreasing or increasing, however. In particular, a (generalized) quasi-aggregative game need be neither a WSTC or a WSTS under this paper's assumptions. The following example illustrates this point.

Example 2 Let $\sigma_{i}\left(s_{-i}\right)=\prod_{j \neq i}\left(1-s_{j}\right)$, and assume that $\tilde{\pi}_{i}(s)=\pi_{i}\left(\sigma_{i}\left(s_{-i}\right), s_{i}\right)$ for all $i$. This game is aggregative as seen by taking $g(s)=-\prod_{i}\left(1-s_{i}\right)$ and $F_{i}\left(x_{-i}, s_{i}\right)=$ $x_{-i}\left(s_{i}-1\right)$ for all $i . F_{i}$ exhibits strictly increasing differences since $D_{x_{-i} s_{i}}^{2} F_{i}\left(x_{-i}, s_{i}\right)=$ $1>0$. Take for example $I=3$ and consider the first player. $\sigma_{1}\left(s_{-1}\right)=\left(1-s_{2}\right)(1-$ $\left.s_{3}\right)$ so $\frac{\partial \sigma_{1}\left(s_{-1}\right)}{\partial s_{2}} \geq 0 \Leftrightarrow s_{3} \geq 1$. So whether the first player's interaction function is increasing, decreasing, or constant in the second player's strategy, depends on the strategy chosen by the third player. Accordingly, under assumption 1, player 1's bestreplies may be increasing, decreasing, or constant in $s_{2}$ depending on $s_{3}$ (and so the game cannot be a WSTC or a WSTS or, for that matter, submodular or supermodular).

The next example shows that in at least one common case, it is necessary to apply a monotone transformation in order for assumption 2 to be satisfied.

Example 3 Take the linear sum case, $g(s)=\sum_{i} s_{i}, \sigma_{i}\left(s_{-i}\right)=\sum_{j \neq i} s_{j}$, and $F_{i}\left(x_{-i}, s_{i}\right)=$ $x_{-i}+s_{i}$ which defines an aggregative game if $\tilde{\pi}_{i}(s)=\pi_{i}\left(\sum_{j \neq i} s_{j}, s_{i}\right)$ for all $i . F_{i}$ does not exhibit strictly increasing differences. However, there obviously exists a monotone transformation such that $h \circ F_{i}$ does satisfies assumption 2 for all $i(h(z)=\exp (z)$, for one).

We end this section with an example of a quasi-aggregative game which does not satisfy assumption 2 .

Example 4 Let each player $i$ 's payoff function depend on $s_{i}$ and $\sigma_{i}\left(s_{-i}\right)=\max _{j \neq i} s_{j}$. This is an aggregative game because we may take $g(s)=\max _{i} s_{i}$ and $F_{i}\left(x_{-i}, s_{i}\right)=$ $\max \left\{x_{-i}, s_{i}\right\}$. Assumption 2 is not satisfied, for this requires that, $\max \left\{\tilde{x}_{-i}, \tilde{s}_{i}\right\}-$ $\max \left\{x_{-i}, \tilde{s}_{i}\right\}>\max \left\{\tilde{x}_{-i}, s_{i}\right\}-\max \left\{x_{-i}, s_{i}\right\}$ whenever $\tilde{x}_{-i}>x_{-i}$ and $\tilde{s}_{i}>s_{i}$ (and equality holds when, say, $s_{i} \geq \tilde{x}_{-i}$ ). It is also clear that no monotonic transformation will resolve this problem.

\subsection{Special Cases}

Each class of solutions to (2)/(4) will yield a special case of a quasi-aggregative game/ generalized quasi-aggregative game. In this section a number of such classes are considered.

\footnotetext{
6 Following Dubey et al. (2006)'s terminology, a generalized aggregative game might then, when the $\sigma_{i}$ 's are increasing or decreasing, be called $a$ WSTS/WSTC with generalized quasi-aggregation (and possibly discontinuous best-reply selections).
} 


\subsubsection{Generalized Symmetric Aggregators (Alos-Ferrer and Ania (2005))}

Let strategy sets be compact subset of the reals, $S_{i} \subseteq \mathbb{R}$, for all $i$, and $\circ: X \times X \rightarrow X$ an increasing and continuous function (here $X$ is an interval in $\mathbb{R}$ large enough to ensure that $S_{i} \subseteq X$ for all $\left.i\right){ }^{7}$ For $n=2,3, \ldots, I$ let,

$$
\circ^{n}\left(s_{1}, \ldots, s_{n}\right)=\circ\left(\circ^{n-1}\left(s_{1}, \ldots, s_{n-1}\right), s_{n}\right) \text { where } \circ^{1}\left(s_{i}\right) \equiv s_{i}
$$

Next assume that every "iterate" $\circ^{n}: X^{n} \rightarrow X$ is a symmetric function, and take as aggregator in a game with $I$ players:

$$
g(s) \equiv o^{I}\left(s_{1}, \ldots, s_{I}\right)
$$

An aggregator constructed in this manner is called a generalized (symmetric) aggregator in Alos-Ferrer and Ania (2005) (the reader is referred to that paper for a long list of examples). By taking $\sigma_{i}\left(s_{-i}\right) \equiv \mathrm{o}^{I-1}\left(s_{-i}\right)$ and $F_{i}\left(x_{-i}, s_{i}\right) \equiv \circ\left(x_{-i}, s_{i}\right)$ for all $i$, it is clear that any such aggregator will satisfy (2). Thus if payoff functions satisfy (1) or the more special form (3), the game will be quasi-aggregative. The assumptions on $\circ$ in Alos-Ferrer and Ania (2005) as reproduced above (o increasing, symmetric, and strategy sets one-dimensional) are all unnecessary for this paper's results. ${ }^{8}$

\subsubsection{Disguised Aggregative Games (Cornes and Hartley (2001)), Generalized Means}

Cornes and Hartley (2001) investigate aggregative games with main focus on the backward reply correspondence $b_{i}(Q)=\left\{s_{i} \in S_{i}: s_{i} \in \tilde{R}_{i}\left(s_{-i}\right), g(s)=Q\right\}$ (cf. Novshek $(1985))$, which the authors also call the replacement correspondence. Cornes and Hartley (2001) prove that if payoffs can be cast in the form (3) for an aggregator $g$ which is twice continuously differentiable and strictly monotonic and the game admits a replacement correspondence, then $g$ must be additively separable. ${ }^{9}$ As explained in section 2.1, all such games will consequently be quasi-aggregative.

Another central element in Cornes and Hartley's paper is the notion that $g(s)$ can be seen as a "statistic" (and they give many examples to this effect). There is potentially a close connection between this observation and the generalized symmetric aggregators of the previous section. Thus if $\circ$ in that construction is a strictly increasing function, $g$ will be a generalized symmetric aggregator if and only if $g(s)=f^{-1}\left(\sum_{i} f\left(s_{i}\right)\right)$ for some strictly increasing function $f .{ }^{10}$ Applying the strictly

\footnotetext{
7 We follow here Schipper (2005) (Definition 3) in taking the domain of o to be $X \times X$ which of course has a restriction to $X_{-i} \times S_{i}$ which then corresponds to $F_{i}$ of our definition 1 .

8 The only condition one must verify is that $F_{i}:=0$ satisfies assumption 2 . This is automatically satisfied, possibly after a monotonic transformation, when $\circ$ is increasing (in particular, assumption 2 is satisfied under the conditions of Alos-Ferrer and Ania (2005)). The recursive definition extends to multidimensional strategy sets in the obvious manner by taking $\circ: X \times T \rightarrow X, X \subseteq \mathbb{R}$ and $S_{i} \subseteq T \subseteq \mathbb{R}^{N}$. If $\circ^{1}$ is not the identity function in one dimension (and chosen suitably in multiple dimensions), $g$ will not be symmetric.

9 A function $g: X \rightarrow \mathbb{R}, X \subseteq \mathbb{R}^{N}$, is additively separable if and only if there exist strictly increasing functions $f_{0}, \ldots, f_{N}: \mathbb{R} \rightarrow \mathbb{R}$, such that $f_{0}(g(s))=\sum_{n} f_{n}\left(s_{n}\right)$ for all $s \in X$. See for example Gorman (1968).

${ }^{10}$ For a detailed proof of this result see the working paper version of this paper (Jensen (2006)), especially Theorem 8 and its corollary.
} 
increasing transformation $z \mapsto f^{-1}\left(I^{-1} f(z)\right)$, we can write this in the more familiar form of a generalized mean (also called a generalized $f$-mean or quasi-arithmetic mean):

$$
g(s)=f^{-1}\left(\frac{1}{I} \sum_{i} f\left(s_{i}\right)\right)
$$

All the standard means are special cases of the generalized mean, including the harmonic mean $\left(f(x)=x^{-1}\right)$, the geometric mean $(f(x)=\ln x)$, and the power means $\left(f(x)=x^{k}\right.$ where $\left.k \in \mathbb{N}\right)$. In particular, a game where each player's payoff function depends only on her own strategy and some generalized mean, is quasi-aggregative. Assumption 2 is automatically satisfied. Indeed, taking $\tilde{g}=\exp [f \circ g]$, one sees that $\tilde{g}(s)=F_{i}\left(s_{i}, \sigma_{i}\left(s_{-i}\right)\right)=\left[\exp \left(f\left(s_{i}\right)\right)\right] \cdot \sigma_{i}\left(s_{-i}\right)$, where $\sigma_{i}\left(s_{-i}\right)=\prod_{j \neq i} \exp \left(f\left(s_{j}\right)\right)$. Since $f$ is strictly increasing, $F_{i}$ exhibits strictly increasing differences in its two arguments. As for assumption 1 (assumption $1^{\prime}$ ), this will hold if each player's payoff function exhibits (strictly) decreasing differences in $s_{i}$ and the opponents' strategies $s_{-i}$.

\subsubsection{Games with Reciprocal Interactions (Dubey et al (2006), Kukushkin (2005))}

$\Gamma$ is a game with reciprocal interactions, if $\tilde{\pi}_{i}(s)=\pi_{i}\left(\sigma_{i}\left(s_{-i}\right), s_{i}\right)$ for all $i$, where the $\sigma_{i}$ 's are real-valued, continuously differentiable interaction functions which satisfy:

$$
\frac{\partial \sigma_{i}\left(s_{-i}\right)}{\partial s_{j}}=\frac{\partial \sigma_{j}\left(s_{-j}\right)}{\partial s_{i}}, \text { for all } i, j \in \mathscr{I} \text { and all } s \in \hat{S}
$$

where $\hat{S}$ is an open, convex subset of $\mathbb{R}^{I}$ which contains $S$.

In a game with reciprocity, the marginal influence through the interaction functions $\sigma_{i}, i \in \mathscr{I}$ is symmetric between any two players. It may be zero (no interaction effect), negative (which can been seen as a congestion effect or negative externality when payoff functions are increasing in $\sigma_{i}$ ), and it may be positive (a spillover effect or positive externality) depending on $s \in S$.

From (8) follows that each $\sigma_{i}\left(s_{-i}\right)$ must be affine in $s_{j}$ when $s_{-j}$ is kept fixed. Hence without loss of generality:

$$
\sigma_{i}\left(s_{-i}\right)=\alpha_{i}+\sum_{m=1}^{I-1} \sum_{j_{1}<\ldots<j_{m}: j_{q} \neq i} \alpha_{i j_{1} \ldots j_{m}} s_{j_{1}} \ldots s_{j_{m}}
$$

where all of the $\alpha$ 's are real constants. Since (8) is required to hold on open intervals, it follows furthermore that $\alpha_{j_{0} j_{1} \ldots j_{m}}=\alpha_{j_{r(0)} j_{r(1)} \ldots j_{r(m)}}$ for any $m>0$ and any permutation $r:\left\{j_{0}, j_{1}, \ldots, j_{m}\right\} \rightarrow\left\{j_{0}, j_{1}, \ldots, j_{m}\right\}$. It is now easily verified that such games are generalized quasi-aggregative upon taking $g(s)=\sum_{m=2}^{I} \sum_{j_{1}<\ldots<j_{m}} \alpha_{j_{1} \ldots j_{m}} s_{j_{1}} \ldots s_{j_{m}}$, $F_{i}\left(x_{-i}, s_{i}\right)=\left(x_{-i}-\alpha_{i}\right) s_{i}$, and $v_{i}\left(s_{-i}\right)=g(s)-\left(\sigma_{i}\left(s_{-i}\right)-\alpha_{i}\right) s_{i}$ (as may be checked, $v_{i}$ is independent of $s_{i}$ as required by (4)). $F_{i}$ clearly exhibits strictly increasing differences (assumption 2) since $D_{s_{i} x_{-i}}^{2} F_{i}\left(x_{-i}, s_{i}\right)=1>0$.

Games with reciprocal interactions were first studied by Dubey et al. (2006) and subsequently generalized by Kukushkin (2005) to the form (9) (the name appears to be due to Kukushkin). 


\subsubsection{Games with Generalized Reciprocal Interactions, Networks}

In one interpretation, games with reciprocal interactions describe symmetric (but potentially very complex) networks. The simplest illustration of this interpretation is when each player's payoff function depends (linearly) on only a subset $N(i) \subseteq \mathscr{I}$ of the other players' strategies. So player $i$ 's payoff function is a function of $s_{i}$ and $\sigma_{i}\left(s_{-i}\right)=\sum_{j \in N(i)} s_{j}$. Reciprocity/symmetry of the network, then amounts to having $j \in N(i) \Leftrightarrow i \in N(j)$ (since then, and only then, will (8) hold for all $i$ ). Because this paper's results do not hinge on the specific functional forms, it is easy to generalize the class so that more complex networks are allowed. Thus a game will still be generalized quasi-aggregative and satisfy assumption 2 if (8) is replaced by any one of the following.

$$
\begin{gathered}
\frac{\partial \sigma_{i}\left(s_{-i}\right)}{\partial s_{j}} / \sigma_{i}\left(s_{-i}\right)=\frac{\partial \sigma_{j}\left(s_{-j}\right)}{\partial s_{i}} / \sigma_{j}\left(s_{-j}\right) \\
\frac{\partial \sigma_{i}\left(s_{-i}\right)}{\partial s_{j}} / s_{i}=\frac{\partial \sigma_{j}\left(s_{-j}\right)}{\partial s_{i}} / s_{j} \\
\frac{\partial \sigma_{i}\left(s_{-i}\right)}{\partial s_{j}} / \frac{\sigma_{i}\left(s_{-i}\right)}{s_{j}}=\frac{\partial \sigma_{j}\left(s_{-j}\right)}{\partial s_{i}} / \frac{\sigma_{j}\left(s_{-j}\right)}{s_{i}}
\end{gathered}
$$

where in (8a) we must assume that every $\sigma_{i}: S_{-i} \rightarrow \mathbb{R}_{++}$, in (8b) that every $s_{i}>0$, and in (8c) we must assume both. Intuitively, (8a) expresses relative reciprocity, (8b) proportional reciprocity, and (8c) reciprocity with respect to elasticities. Needless to say, either one of these may be the more realistic one depending on the concrete application. As mentioned, assumption 2 will also be satisfied. ${ }^{11}$

The "trick" used above to generalize a class of games by transforming one or more of the involved functionals, should not be confused with transformations of the strategies. Crucially, it may be that assumption 2 is satisfied before a transformation but not after (and so this paper's results do not apply). In this respect there is nothing trivial about such transformation based results.

\subsubsection{Aggregators with Weakly Separable Derivatives, the Variance and Other Summary Statistics}

Frequently one has a game where payoff functions take the form (3) for a known aggregator $g$. In order for this paper's results to apply, it must then be established that the game is generalized quasi-aggregative (definition 2). In this case, the functions $\sigma_{i}, F_{i}$, and $v_{i}$, play a purely auxiliary role. When $g$ is continuously differentiable and $S_{i} \subseteq \mathbb{R}$ for all $i$, the game will be generalized quasi-aggregative provided that $g$ 's first derivatives are weakly separable w.r.t. each of the partitions $\left\{s_{i}, s_{-i}\right\}$. Precisely, there

\footnotetext{
11 In the first case, transform the interaction functions, $\tilde{\sigma}_{i}\left(s_{-i}\right)=\log \sigma_{i}\left(s_{-i}\right)$ to conclude that the game is generalized aggregative with $F_{i}\left(x_{-i}, s_{i}\right)=\log \left(x_{-i}\right) s_{i}+v_{i}\left(s_{-i}\right)$ (which satisfies assumption 2 since $\left.D_{x_{-i} s_{i}}^{2} F_{i}\left(x_{-i}, s_{i}\right)=\frac{1}{x_{-i}}>0\right)$. In the second case transform the strategies, $F_{i}\left(x_{-i}, s_{i}\right)=x_{-i} \log \left(s_{i}\right)+v_{i}\left(s_{-i}\right)$, and in the third transform both the interaction functions and the strategies, $F_{i}\left(x_{-i}, s_{i}\right)=\log \left(x_{-i}\right) \log \left(s_{i}\right)+$ $v_{i}\left(s_{-i}\right)$. In either case $F_{i}$ is smooth and satisfies assumption 2.
} 
must for each agent $i \in \mathscr{I}$ exist functions $f_{i}$ and $\sigma_{i}$ such that: ${ }^{12}$

$$
\frac{\partial g(s)}{\partial s_{i}}=f_{i}\left(\sigma_{i}\left(s_{-i}\right), s_{i}\right) \text { for all } s \in S
$$

Directly verifying (10) is sometimes the easiest way to establish that a given game is generalized quasi-aggregative. As an example, imagine that each payoff function depends only on the player's own strategy and the variance of all players' strategies (for an extension see section 5). That is to say, assume that (3) holds with,

$$
g(s)=\operatorname{Var}(s)=n^{-1}\left(\sum_{i} s_{i}^{2}\right)-\left(n^{-1}\left(\sum_{i} s_{i}\right)\right)^{2}
$$

Then $\frac{\partial g(s)}{\partial s_{i}}=2 n^{-1}\left(1-n^{-1}\right) s_{i}-2 n^{-1}\left(\sum_{j \neq i} s_{j}\right)$. So taking $\sigma_{i}\left(s_{-i}\right)=\sum_{j \neq i} s_{j},(10)$ is satisfied with $f_{i}\left(x_{-i}, s_{i}\right)=2 n^{-1}\left(1-n^{-1}\right) s_{i}-2 n^{-1} x_{-i}$. We conclude that such games are generalized quasi-aggregative. In addition, $F_{i}$ exhibits strictly decreasing differences since $f_{i}\left(x_{-i}, s_{i}\right)$ is strictly decreasing in $x_{-i}$. Hence $F_{i}$ satisfies assumption 2 (with the affine transformation $z \mapsto h(z)=-z$ ). As a second (admittedly somewhat fancy) example assume that $g(s)$ equals the sample mean of a log-normal distribution: $E(s)=\exp \left(\mu+0.5 \sigma^{2}\right)$ where $\mu$ is the average and $\sigma^{2}$ is the variance of the logarithm of the strategy vector. We would in this case have:

$$
g(s)=\exp \left[n^{-1}\left(\sum_{i} \log \left(s_{i}\right)\right)+0.5 n^{-1}\left(\sum_{i}\left(\log \left(s_{i}\right)\right)^{2}\right)-\left(n^{-1}\left(\sum_{i} \log \left(s_{i}\right)\right)\right)^{2}\right]
$$

As may be checked via (10), this is generalized quasi-aggregative with $\sigma_{i}\left(s_{-i}\right)=$ $\sum_{j \neq i} \log \left(s_{j}\right)$ and $F_{i}\left(s_{i}, x_{-i}\right)=n s_{i}^{-1}\left(\log \left(s_{i}\right) s_{i}^{-1}-2\left(\log \left(s_{i}\right)+x_{-i}\right)\right)$.

\section{Aggregative Games and Best-Reply Potentials}

In this section is shown that if assumptions $1^{\prime}$ and 2 are satisfied, then a generalized quasi-aggregative game is a best-reply potential game as defined by Voorneveld (2000). If assumption 1 is imposed rather than the (stronger) assumption $1^{\prime}$, the game is a (best-reply) pseudo-potential game as defined in Dubey et al. (2006). The relevant definitions are as follows:

Definition 3 (Best-Reply Potential Games) $\left(\tilde{R}_{i}, S_{i}\right)_{i \in \mathscr{I}}$ is a best-reply potential game if there exists a function $P: S \rightarrow \mathbb{R}$ (the potential) such that for all $i \in \mathscr{I}$ and $s_{-i} \in S_{-i}$ :

$$
\tilde{R}_{i}\left(s_{-i}\right)=\arg \max _{s_{i} \in S_{i}} P\left(s_{i}, s_{-i}\right)
$$

It is a best-reply pseudo-potential game if (13) is weakened to:

$$
\tilde{R}_{i}\left(s_{-i}\right) \supseteq \arg \max _{s_{i} \in S_{i}} P\left(s_{i}, s_{-i}\right)
$$

\footnotetext{
12 To see this, integrate (10) to get $g(s)=\int_{-\infty}^{s_{i}} f_{i}\left(\sigma_{i}\left(s_{-i}\right), \tau\right) d \tau+v_{i}\left(s_{-i}\right) \equiv F_{i}\left(\sigma_{i}\left(s_{-i}\right), s_{i}\right)+v_{i}\left(s_{-i}\right)$ which is exactly (4).
} 
The previous definition of a best-reply potential game is identical to that found in Voorneveld (2000). Dubey et al. (2006) as part of their definition of a pseudopotential game include the assumption that the potential must be a continuous function. The potential functions of the following theorem will in general only be upper semi-continuous. As explained below, a continuous pseudo-potential function can, however, be found if a continuous decreasing best-reply selection exists.

Theorem 1 (Quasi-Aggregative Games are Pseudo-potential Games) Let $\Gamma$ be a (generalized) quasi-aggregative game with compact strategy sets and upper semicontinuous payoff functions. Then if assumptions $1^{\prime}$ and 2 are satisfied, the game is a best-reply potential game. If assumptions 1 and 2 are satisfied, the game is a bestreply pseudo-potential game. In either case, associated potential functions may be found which are upper semi-continuous.

Proof: Section 6.1.

Remark 31 Theorem 1 remains valid if instead of assumptions 1, it it assumed that each $R_{i}$ has an increasing selection. So, like Theorem 1 in Dubey et al. (2006), the result applies equally to games of weak strategic substitutes or complements. More generally, the result remains valid under any monotonic transformation of the involved functions (see the discussion following assumption 2).

Theorem 1 parallels the main result in Dubey et al. (2006) who establish that an aggregative game is a pseudo-potential game if (i) aggregation is linear, and (ii) the game has a continuous best-reply selection which is either increasing or decreasing (strategic substitutes or complements). As mentioned above, the potential function determined by Dubey et al. (2006) is a continuous function under these assumptions. If the best-reply selection is not continuous, Dubey et al. (2006)'s argument does not apply, and the question answered in theorem 1 is left by the authors as an open question (see Dubey et al. (2006), beginning of section 6). It should in this connection be mentioned that if a continuous best-reply selection exists in the present setting, a continuous potential function can be found too (see remark 61 in the proof). Thus theorem 1 both generalizes and strengthens Dubey et al. (2006). The first part of theorem 1 (where assumption $1^{\prime}$ is invoked) is also closely related to the main result of Kukushkin (2005) who proves the existence of a Cournot potential for a generalized class of games with reciprocal interactions along the lines of that considered in section 5 of Dubey et al. (2006) (see section 2.3.3 for the details). The existence of a Cournot potential does not imply that the game is a best-reply potential game, however (it is a necessary but not a sufficient condition).

Whether or not strategy sets are convex, and whether or not payoff functions are quasi-concave (none of which has been assumed anywhere), it is an immediate consequence of theorem 1 that a pure strategy Nash equilibrium will exist.

Corollary 1 (Existence of PSNE) Let $\Gamma$ be a (generalized) quasi-aggregative game with compact strategy sets and upper semi-continuous payoff functions. Then if assumptions 1 and 2 are satisfied, there exists a PSNE. 
Proof: By assumption 1, each $R_{i}$ has a decreasing selection $r_{i}: X_{-i} \rightarrow S_{i}$. Replace each $R_{i}$ with the sectioning of the closure of the graph of $r_{i} .{ }^{13}$ The resulting subcorrespondence has a closed graph and satisfies assumption $1^{\prime}$. For the existence of a PSNE it is sufficient to consider such a subcorrespondence: If $\hat{R}_{i}: X_{-i} \rightarrow 2^{S_{i}}$ is a subcorrespondence of $R_{i}, \hat{R}_{i}\left(x_{-i}\right) \subseteq R_{i}\left(x_{-i}\right)$ for all $x_{-i} \in X_{-i}$, then it is obviously true that if $s^{*} \in \hat{R}_{i}\left(\sigma_{i}\left(s_{-i}^{*}\right)\right)$ for all $i$, then $s^{*} \in R_{i}\left(\sigma_{i}\left(s_{-i}^{*}\right)\right)$ for all $i$, and so it is a PSNE. By theorem 1, P may be taken to be upper semi-continuous, hence it takes a maximum on the compact set $S$. Any maximizer is trivially a PSNE.

Corollary 1 contains as special cases the existence theorems of Dubey et al. (2006),

Kukushkin (1994), Kukushkin (2005), and Novshek (1985). It is the first to answer in the affirmative a question raised implicitly in Alos-Ferrer and Ania (2005), namely whether a PSNE will exist under that paper's notion of aggregation. Since this notion of aggregation is so widely studied within evolutionary game theory (see $e . g$. Schipper (2005) and references therein), our affirmative answer would seem to be of some value. As explained in section 2, a quasi-aggregative game will be a game of strategic complementarities if the interaction functions $\sigma_{i}: S_{-i} \rightarrow \mathbb{R}$ are coordinatewise decreasing. In this case, existence of a PSNE follows from Tarski's fixed point theorem provided that $S$ is a complete lattice (and as is well known, any aggregation assumptions are superfluous from the point of view of existence of PSNE). If $S$ is not a complete lattice (which clearly is possible because we allow for multidimensional strategy sets), the above corollary still applies even though Tarski's fixed point theorem does not.

A referee has asked me to address the general relationship between best-reply potential games and quasi-aggregative games. For the following denote the set of generalized quasi-aggregative games by $\Gamma^{G Q A}$, the set of quasi-aggregative games by $\Gamma^{Q A}$, the set of potential games by $\Gamma^{P}$, the set of best-reply potential games by $\Gamma^{B P}$, and the set of best-reply pseudo-potential games by $\Gamma^{P B P}$. Clearly $\Gamma^{Q A} \subset \Gamma^{G Q A}$ and $\Gamma^{P} \subset \Gamma^{B P} \subset \Gamma^{P B P}$, and by theorem $1, \Gamma^{Q A} \cap \Gamma^{P B P} \neq \emptyset$. The following two examples show that if either assumption 1 or assumption 2 is violated, a quasi-aggregative game may fail to have a PSNE. Since any game in $\Gamma^{P B P}$ has a PSNE, it follows in particular that $\Gamma^{Q A} \nsubseteq \Gamma^{P B P}$ (whence $\Gamma^{G Q A} \nsubseteq \Gamma^{P B P}, \Gamma^{Q A} \nsubseteq \Gamma^{B P}, \Gamma^{G Q A} \nsubseteq \Gamma^{B P}, \Gamma^{Q A} \nsubseteq \Gamma^{P}$, and $\left.\Gamma^{G Q A} \nsubseteq \Gamma^{P}\right)$.

Example 5 Take $I=2, S_{1}=S_{2}=[0,1]$, and consider the (upper hemi-continuous) best-reply correspondences, $\tilde{R}_{2}\left(s_{1}\right)=\left\{1-s_{1}\right\}$, for $s_{1} \in[0,1]$, and $\tilde{R}_{1}\left(s_{2}\right)=\left\{0.5-s_{2}\right\}$ for $s_{2} \in[0,0.5), \tilde{R}_{1}(0.5)=\{0,1\}$, and $\tilde{R}_{1}\left(s_{2}\right)=\left\{1.5-s_{2}\right\}$, for $s_{2} \in(0.5,1]$. This game is quasi-aggregative and satisfies assumption $2 .{ }^{14}$ It is easy to verify that a

\footnotetext{
13 The graph of $r_{i}$ is $\operatorname{Graph}\left(\hat{r}_{i}\right)=\left\{\left(x_{-i}, s_{i}\right): s_{i}=\hat{r}_{i}\left(x_{-i}\right)\right\}$. Since $R_{i}$ has a closed graph, $\mathrm{Cl}\left(\operatorname{Graph}\left(\hat{r}_{i}\right)\right) \subseteq$ $\operatorname{Graph}\left(R_{i}\right)=\left\{\left(x_{-i}, s_{i}\right): s_{i} \in R_{i}\left(x_{-i}\right)\right\}$ (here $\mathrm{Cl}$ denotes the closure). Consequently, the sectioning of the closure, $\hat{R}_{i}\left(x_{-i}\right)=\left\{s_{i} \in S_{i}:\left(s_{i}, x_{-i}\right) \in \mathrm{Cl}\left(\operatorname{Graph}\left(\hat{r}_{i}\right)\right)\right\}$, is a subcorrespondence of $R_{i}$.

14 Take $\sigma_{1}\left(s_{2}\right)=s_{2}, \sigma_{2}\left(s_{1}\right)=s_{1}, g(s)=\exp \left(s_{1}+s_{2}\right), F_{1}\left(s_{1}, x_{-1}\right)=\exp \left(s_{1}+x_{-1}\right)$, and $F_{2}\left(s_{2}, x_{-2}\right)=$ $\exp \left(s_{2}+x_{-2}\right)$. Needless to say, payoff functions that yield these best-reply correspondences may be produced so that (1) is satisfied.
} 
PSNE does not exist (depict the correspondences in a diagram with $s_{1}$ on the first axis and $s_{2}$ on the second axis).

Example $6^{15}$ Let $S_{1}=S_{2}=S_{3}=[0,1]$ and consider the best-reply correspondences:

$$
\begin{aligned}
& \tilde{R}_{1}\left(s_{2}, s_{3}\right)=\left\{\begin{array}{l}
\{1\} \text { if } s_{2}<0.5 \\
\{0,1\} \text { if } s_{2}=0.5 \\
\{0\} \text { if } s_{2}>0.5
\end{array}\right. \\
& \tilde{R}_{2}\left(s_{1}, s_{3}\right)=\left\{\begin{array}{l}
\{1\} \text { if } s_{3}<0.5 \\
\{0,1\} \text { if } s_{3}=0.5 \\
\{0\} \text { if } s_{3}>0.5
\end{array}\right. \\
& \tilde{R}_{3}\left(s_{1}, s_{2}\right)=\left\{\begin{array}{l}
\{1\} \text { if } s_{1}<0.5 \\
\{0,1\} \text { if } s_{1}=0.5 \\
\{0\} \text { if } s_{1}>0.5
\end{array}\right.
\end{aligned}
$$

Taking $\sigma_{1}\left(s_{-1}\right)=s_{2}, \sigma_{2}\left(s_{-2}\right)=s_{3}$, and $\sigma_{3}\left(s_{-3}\right)=s_{1}$, we can write $\tilde{R}_{1}\left(s_{-1}\right)=$ $R_{1}\left(\sigma\left(s_{-1}\right)\right), \tilde{R}_{2}\left(s_{-2}\right)=R_{2}\left(\sigma\left(s_{-2}\right)\right)$, and $\tilde{R}_{3}\left(s_{-3}\right)=R_{3}\left(\sigma\left(s_{-3}\right)\right)$. Assumption $1^{\prime}$ is clearly satisfied $\left(R_{1}, R_{2}\right.$, and $R_{3}$ are strictly decreasing), and one easily constructs "supporting" payoff functions in the form (1). (2) is satisfied with $g(s)=F_{i}\left(s_{i}, x_{-i}\right)=$ 0 for all $i$, say. No equilibrium exists: If $s_{1}=1$ then $s_{2} \leq 0.5$, so $s_{2}=0$, but then $s_{3}=1$ and so $s_{1}=0$ (likewise if $s_{1}=0$ ).

All best-reply potential games can be cast in the form (3) and are in this sense "aggregative" unless further conditions are imposed. Indeed, if a best-reply potential game has potential $P: S \rightarrow \mathbb{R}$, then (3) trivially holds for all $i$ by taking $\hat{\pi}_{i}\left(s_{i}, g(s)\right)=$ $g(s)$ and $g:=P$. Not all (pseudo) best-reply potential games are generalized quasiaggregative, however. The following modification of example 1 establishes this fact by showing that $\Gamma^{P} \nsubseteq \Gamma^{G Q A}$ (whence $\Gamma^{B P} \nsubseteq \Gamma^{G Q A}, \Gamma^{P B P} \nsubseteq \Gamma^{G Q A}, \Gamma^{B P} \nsubseteq \Gamma^{Q A}$, and $\left.\Gamma^{P B P} \nsubseteq \Gamma^{Q A}\right)$. Combining with the examples above, we are led to conclude that best-reply potential games and (generalized) quasi-aggregative games are in general distinct classes of games.

Example 7 Consider a three-player game with the payoff functions $\tilde{\pi}_{1}(s)=\gamma_{1} s_{1}$ $\frac{1}{2} s_{1}^{2} s_{2}-\frac{1}{3} s_{1}^{3} s_{3}-s_{1} s_{2}, \tilde{\pi}_{2}(s)=\gamma_{2} s_{2}-\frac{1}{2} s_{1}^{2} s_{2}-s_{1} s_{2}-s_{2}^{2}, \tilde{\pi}_{3}(s)=\gamma_{3} s_{3}-\frac{1}{3} s_{1}^{3} s_{3}-s_{3}^{2}$ (here $S_{i} \subseteq \mathbb{R}, i=1,2,3$, and $\left.\gamma_{1}, \gamma_{2}, \gamma_{3}>0\right)$. These payoff functions are strictly concave in players' own strategies and solutions will be interior if the $\gamma$ 's are sufficiently large. So there is nothing "perverse" about this example. Clearly, this is a potential game with $P(s)=\gamma_{1} s_{1}+\gamma_{2} s_{2}+\gamma_{3} s_{3}-\frac{1}{2} s_{1}^{2} s_{2}-\frac{1}{3} s_{1}^{3} s_{3}-s_{1} s_{2}-s_{2}^{2}-s_{3}^{2}$. But it is not generalized quasi-aggregative, since (1) cannot hold for any choice of functions $\pi_{i}, \sigma_{i}, i=1,2,3$. The argument runs exactly as in example 1: If (1) were to hold for player $i=1$, it would follow that $(*) D_{s_{1}} P(s)=D_{s_{1}} \pi_{i}\left(\sigma_{1}\left(s_{-1}\right), s_{1}\right)$. (*) implies that for all $\hat{s}_{1} \in S_{1}$, $D_{s_{1}} P\left(\hat{s}_{1}, \cdot, \cdot\right)$ is constant on any of the sets $\Delta_{1}\left(\alpha, s_{1}\right)=\left\{\left(s_{2}, s_{3}\right) \in S_{2} \times S_{3}: D_{s_{1}} P(s)=\right.$ $\alpha\}=\left\{\left(s_{2}, s_{3}\right): \gamma_{1}-s_{1} s_{2}-s_{1}^{2} s_{3}-s_{2}=\alpha\right\}, s_{1} \in S_{1}$ and $\alpha \in P(S)$. But this is false in general.

15 This example is due to Nikolai Kukushkin (personal communication). 
4 Best-Reply Dynamics

We next turn to the dynamic properties of the equilibrium set. The dynamic concept to be studied dates back to Cournot, who considered a duopoly where the firms sequentially update their best-replies and proved convergence to a PSNE (Cournot (1838)). Among the results of this section is the observation that, roughly, Cournot's observations carry over to arbitrary generalized quasi-aggregative games under assumptions similar to those of Cournot.

A sequence $\mathbf{s}=\left(s^{0}, s^{1}, s^{2}, \ldots\right)$ in $S$ is a sequential improvement path if each player moves in turn, to a strictly preferred strategy if one exist and if not, stays with the previous strategy. Precisely, for all $t$ there is an $i$ such that $s_{-i}^{t}=s_{-i}^{t+1}$, and either (i) $s_{i}^{t+1} \in R_{i}\left(\sigma_{i}\left(s_{i-1}^{t}\right)\right), s_{i}^{t} \notin R_{i}\left(\sigma_{i}\left(s_{-i}^{t}\right)\right)$, or else (ii) $s_{i}^{t+1}=s_{i}^{t} \in R_{i}\left(\sigma_{i}\left(s_{-i}^{t}\right)\right)$. The path is admissible if whenever $I$ successive periods have passed all $I$ different players have moved (possibly to a previous strategy, $s_{i}^{t+1}=s_{i}^{t} \in R_{i}\left(\sigma_{i}\left(s_{i-1}^{t}\right)\right)$ ). Admissibility ensures that all of the players actually get to move. If this is not required, there is nothing to be said in general about improvement paths' dynamics. ${ }^{16}$

For $\left(s^{t}\right)$ a sequence in $S$, say that $\left(s^{t}\right)$ converges to the set $A \subseteq S$ if the limit point of any convergent subsequence lies in $A \cdot{ }^{17}$ An indifference path is a non-constant, infinite path where players never move to a strictly preferred best-reply: $\left\{s_{i}^{t+1}, s_{i}^{t}\right\} \subseteq$ $R_{i}\left(\sigma_{i}\left(s_{-i}^{t}\right)\right), s_{-i}^{t}=s_{-i}^{t+1}$, all $t$.

Theorem 2 Consider a (generalized) quasi-aggregative game $\Gamma$ with compact strategy sets and upper semi-continuous payoff functions, satisfying assumptions $1^{\prime}$ and 2. Furthermore assume that $\Gamma$ does not have any indifference paths. Then any admissible sequential improvement path converges to a set of PSNEs. If the PSNE is unique it is consequently Cournot stable: Every admissible sequential improvement path converges to it.

Proof: Section 6.2.

When best-replies are single-valued (e.g., payoff functions are strictly quasiconcave), a player must either move to a strictly preferred best-reply or remain with the previous strategy. This clearly rules out indifference paths. So the conclusion of theorem 2 will apply to any (generalized) quasi-aggregative game with one-dimensional compact strategy sets and upper semi-continuous strictly quasi-concave payoff functions, satisfying assumptions 1 and $2 .{ }^{18}$

If assumption 1 is strengthened, we can say more. Assumption $1^{\prime}$ can be written $\tilde{x}_{-i}>x_{-i} \Rightarrow \tilde{s}_{i} \leq s_{i}$ for all $\tilde{s}_{i} \in R_{i}\left(\tilde{x}_{-i}\right)$ and $s_{i} \in R_{i}\left(x_{-i}\right)$. The following strengthening

\footnotetext{
16 The trivial counter-example is when the same player moves at all dates. More generally, it must be ruled out that the same subset of players moves at all dates. With for example three players, one could imagine that only two of them moved at all dates, possibly changing to a strictly preferred strategy at all dates. The process might converge, but only to a PSNE by coincidence because the third player never moves.

17 Recall that the positive limit set of a sequence $\mathbf{s}$ is the set of convergent subsequences of $\mathbf{s}, \Omega(\mathbf{s})=$ $\left\{s \in S: s=\lim _{k \rightarrow \infty} s^{k},\left(s^{k}\right)\right.$ a subsequence of $\left.\mathbf{s}\right\}$ (see e.g. Agerwal (2000)). That $\mathbf{s}$ converges to a set $A$ is thus equivalent to saying that $\Omega(\mathbf{s}) \subseteq A$.

18 This result can be found in Jensen (2007b).
} 
rules out any flat segments in the best-reply correspondences.

Assumption 1" Each of the correspondences $R_{i}: X_{-i} \rightarrow 2^{S_{i}}$ satisfies $\tilde{x}_{-i}>x_{-i} \Rightarrow$ $\tilde{s_{i}}<s_{i}$ for all $\tilde{s}_{i} \in R_{i}\left(\tilde{x}_{-i}\right)$ and $s_{i} \in R_{i}\left(x_{-i}\right)$.

Remark 41 Amir (1996) establishes sufficient conditions for assumption $1^{\prime \prime}$ to be satisfied (so called "strict comparative statics conditions"). What is needed is: (i) That agent $i$ 's best-replies are always in the interior of $S_{i}$, (ii) That strategy sets are locally convex, and (iii) That $D_{x_{-i}} \pi_{i}\left(s_{i}, x_{-i}\right)$ exists and is strictly decreasing in $s_{i}$ (if $D_{x_{-i} s_{i}}^{2} \pi_{i}\left(s_{i}, x_{-i}\right) \ll 0$ this will be the case). See also Edlin and Shannon (1998) who present an alternative (and weaker) set of conditions.

Say that a quasi-aggregative game is separable if the aggregator $g$ is a separable function. An indifference cycle of period $k$ is an indifference path where each player moves in a cycle, $\left(s_{i}^{1}, s_{i}^{2}, \ldots, s_{i}^{k}, s_{i}^{1}, s_{i}^{2}, \ldots\right)$ (if $k=1$, the indifference path is thus trivial). For the next result, recall that $I$ denotes the number of players.

Proposition 1 Let $\Gamma$ be a separable quasi-aggregative game with compact strategy sets, upper semi-continuous payoff functions, satisfying assumptions $1^{\prime \prime}$ and 2 . Then any sequential improvement path is attracted to a set of indifference cycles of period $k \leq I-1$.

Proof: Under assumption $1^{\prime \prime}, s_{i}^{t}, s_{i}^{t+1} \in R_{i}\left(\sigma_{i}\right)$, where $\sigma_{i} \in \mathbb{R}$ are constants. By definition of a sequential path, $\sigma_{i}=\sigma_{i}\left(s_{-i}^{t}\right)$ for all $i$ and $t$. Since $g$ is separable, there is no loss of generality in taking $\sigma_{i}\left(s_{-i}\right)=\sum_{j \neq i} s_{j}$ in the following. Let $s^{0}$ be the initial point of an indifference path and assume without loss of generality that players move in the sequence $\{1,2, \ldots, I, 1,2, \ldots\}$. So at date $t=1$ player 1 moves and at date $t=I$ the last player moves. Write $s_{i}^{\tau}$ for the strategy which player i moves to within the time interval $t \in\{\tau I+1, \ldots, \tau I+I\}$. With this notation, it must clearly hold that $\sigma_{i}+s_{i}^{\tau}=\sigma_{i+1}+s_{i+1}^{\tau-1}, i=1, \ldots, I-1$, and $\sigma_{I}+s_{I}^{\tau}=\sigma_{1}+s_{1}^{\tau}$. The conclusion of the theorem now follows: To illustrate for player $i=1: \sigma_{1}+s_{1}^{\tau}=\sigma_{2}+s_{2}^{\tau-1}=\sigma_{3}+s_{3}^{\tau-2}=$ $\ldots=\sigma_{1}+s_{1}^{\tau-(I-1)}$. So if player 1 chooses $s_{1}^{\prime}$ at some point, she will return to $s_{1}^{\prime}$ again after (at most) I- 1 moves, and so forth.

So under assumption $1^{\prime \prime}$, sequential improvement paths are attracted to cycles, possibly trivial ones (i.e., infinite repetitions of a PSNE). Notice as an immediate corollary that in a two-player game such as Cournot duopoly, the conclusion of theorem 2 must then hold (when $I=2$, the indifference cycles must be of period $k=1$, i.e., they must be trivial).

\section{Two Applications}

This section briefly considers two concrete applications. In either case, the aggregation concepts considered in Alos-Ferrer and Ania (2005), Dubey et al. (2006), or Kukushkin (2005) do not apply. In particular, the observation that the games considered will be best-reply (pseudo) potential games could not have been reached without 
the notion of quasi-aggregative games. In the first example, strategy sets are multidimensional. In the second example, strategy sets are one-dimensional but the aggregator is neither separable, generalized symmetric, or linear in each player's strategies.

\subsection{A Teamwork Project with Multiple Tasks}

$I$ agents must each complete a sequence of $T_{i} \in \mathbb{N}$ tasks. If an agent is successful in each part of the sequence, her individual project succeeds. If everyone is successful in their individual projects, the agents' common project succeeds. Otherwise it fails. $s_{i}^{n}$ is the probability that agent $i$ succeeds in task number $n \in\left\{1, \ldots, T_{i}\right\}$. $s_{i}=\left(s_{i}^{1}, \ldots, s_{i}^{T_{i}}\right) \subseteq S_{i}=[0,1]^{T_{i}}$ is agent $i$ 's strategic variable. Intuitively, the agent can choose the level of effort devoted to each of the $T_{i}$ tasks (see Dubey et al. (2006), Example 1 for a similar set-up). Payoff functions depend on own strategies and the (expected) probability of joint success. So we have, $\tilde{\pi}_{i}(s)=\pi_{i}\left(s_{i}, g(s)\right)$, all $i \in \mathscr{I}$, where $g(s)=\prod_{j \in \mathscr{I}} \prod_{n \in\left\{1, \ldots, T_{j}\right\}} s_{j}^{n}$ (the probability that the common project succeeds). The game is quasi-aggregative (take $F_{i}\left(x_{-i}, s_{i}\right)=\left(\prod_{n \in\left\{1, \ldots, T_{i}\right\}} s_{i}^{n}\right) x_{-i}$ and $\left.\sigma_{i}\left(s_{-i}\right)=\prod_{j \neq i} \prod_{n \in\left\{1, \ldots, T_{j}\right\}} s_{j}^{n}\right)$. Assumption 2 will hold if boundary conditions are placed on payoff functions so that no agent "deliberately fails his project" (i.e., chooses some $s_{i}^{n}=0$ ). ${ }^{19}$ Assumption 1 (assumption $1^{\prime}$ ) will be satisfied if $\tilde{\pi}_{i}$ exhibits (strictly) decreasing or (strictly) increasing differences in $s_{i}$ and $s_{-i}$ (which is the usual definition of a game of strategic substitutes/complements). ${ }^{20}$ We conclude that such games are pseudo-potential games, and best-reply potential games if assumption $1^{\prime}$ holds (theorem 1).

\subsection{Mean-Variance Utility}

An interesting example of a generalized quasi-aggregative game arises when payoffs depend on a linear combination of the mean and variance. Specifically, let $\tilde{\pi}_{i}(s)=$ $\hat{\pi}_{i}\left(s_{i}, g(s)\right)$, all $i$, where,

$$
g(s)=\alpha \operatorname{Mean}(s)+\beta \operatorname{Var}(s)=\alpha I^{-1} \sum_{i} s_{i}+\beta I^{-1}\left(\sum_{i} s_{i}^{2}\right)-\beta\left(I^{-1}\left(\sum_{i} s_{i}\right)\right)^{2}
$$

So we are in the setting of (3) and must find suitable functions $\sigma_{i}, F_{i}, v_{i}, i \in \mathscr{I}$ such that (4) holds. This will be the case with $\sigma_{i}\left(s_{-i}\right)=\sum_{j \neq i} s_{j}, F_{i}\left(s_{i}, x_{-i}\right)=\alpha I^{-1} s_{i}+$ $\beta I^{-1} s_{i}^{2}-\beta I^{-2}\left(s_{i}+x_{-i}\right)^{2}$, and $v_{i}\left(s_{-i}\right)=\alpha I^{-1} \sum_{j \neq i} s_{j}+\beta I^{-1}\left(\sum_{j \neq i} s_{j}^{2}\right)$. Assume $\beta \neq 0$ (if $\beta=0$, (15) reduces to a linear aggregator). Assumption 2 is satisfied because $D_{s_{i} x_{-i}}^{2} F_{i}=-2 \beta I^{-2}$, which is strictly greater than zero if $\beta<0$ (if $\beta>0$, apply the linear transformation $F_{i} \mapsto-F_{i}$ ). Because $\sigma_{i}$ is linear, assumption 1 will hold provided

\footnotetext{
19 Simply observe that, $D_{x_{-i} s_{i}}^{2} F_{i}\left(x_{-i}, s_{i}\right)=\left(\prod_{n \neq 1} s_{i}^{n}, \ldots, \prod_{n \neq T_{i}} s_{i}^{n}\right) \gg 0$ for $s_{i} \in(0,1]^{T_{i}}$.

20 This is sufficient, but not necessary even for $\pi_{i}$ to exhibit decreasing/increasing differences in $s_{i}$ and $x_{-i}$.
} 
that this is a game of strategic substitutes (in the usual sense). Topkis' condition for this reads:

$$
\frac{\partial^{2} \pi_{i}\left(s_{i}, g(s)\right)}{\partial s_{i} \partial s_{j}}=D_{12} \pi_{i} \frac{\partial g(s)}{\partial s_{i}}+D_{22} \pi_{i} \frac{\partial g(s)}{\partial s_{i}} \frac{\partial g(s)}{\partial s_{j}}+D_{2} \pi_{i} \frac{\partial^{2} g(s)}{\partial s_{i} \partial s_{j}} \leq 0
$$

where $\frac{\partial g(s)}{\partial s_{i}}=\alpha I^{-1}+2 I^{-1} \beta\left[s_{i}-\frac{\sum_{j} s_{j}}{I}\right]$ and $\frac{\partial^{2} g(s)}{\partial s_{i} \partial s_{j}}=-2 \beta I^{-2}$. If this is met (which obviously requires additional structure on $g$ and/or payoff functions), we conclude as in the previous example.

\section{Proofs}

\subsection{Proof of Theorem 1}

Define two binary relations: The weak improvement relation, $\tilde{s} \succeq s \Leftrightarrow \exists i \in \mathscr{I}$ s.t. $\left[\tilde{s}_{-i}=s_{-i}\right.$, and $\left.\tilde{s}_{i} \in R_{i}\left(\sigma_{i}\left(s_{-i}\right)\right)\right]$, and the improvement relation, $\tilde{s} \succ s \Leftrightarrow[\tilde{s} \succeq s$ and $\left.s_{i} \notin R_{i}\left(\sigma_{i}\left(s_{-i}\right)\right)\right]$. The following lemma shows how these improvement relations are related to best-reply potential games. ${ }^{21}$

Lemma 1 The game $\left(\tilde{R}_{i}, S_{i}\right)_{i \in \mathscr{I}}$ is a best-reply potential game if and only if there exists a real-valued function, $P: S \rightarrow \mathbb{R}$ such that:

$$
\tilde{s} \succeq s \Rightarrow P(\tilde{s}) \geq P(s)
$$

and

$$
\tilde{s} \succ s \Rightarrow P(\tilde{s})>P(s)
$$

The game is a best-reply pseudo-potential game if and only if there exist subcorrespondences, $\hat{R}_{i}: X_{-i} \rightarrow 2^{S_{i}}, \hat{R}_{i}\left(x_{-i}\right) \subseteq R_{i}\left(x_{-i}\right)$, all $x_{-i}$, such that the game that arises when every $R_{i}$ is replaced with $\hat{R}_{i}$ is a best-reply potential game.

Proof: The second claim concerning the pseudo-potential games case is a direct consequence of the definitions and the first statement. So we only need to prove that $\left(\tilde{R}_{i}, S_{i}\right)_{i \in \mathscr{I}}$ is a best-reply potential game if and only if (17)-(18) hold. The "only if" part is trivial: A best-reply potential must clearly satisfy these two conditions. As for the converse, fix any $s_{-i} \in S_{-i}$, take $\tilde{s}_{i} \in \arg \max _{s_{i} \in S_{i}} P\left(s_{i}, s_{-i}\right)$ and assume, by way of contradiction, that $\tilde{s}_{i} \notin \tilde{R}_{i}\left(s_{-i}\right)$. But by (18), $P\left(\tilde{s}_{i}, s_{-i}\right)<P(s)$ for any $s_{i} \in \tilde{R}_{i}\left(s_{-i}\right)$. A contradiction. Next take $\tilde{s}_{i} \in \tilde{R}\left(s_{-i}\right)$ and assume that $\tilde{s_{i}} \notin \arg \max _{s_{i} \in S_{i}} P\left(s_{i}, s_{-i}\right)$, i.e., $P\left(s_{i}, s_{-i}\right)>P\left(\tilde{s}_{i}, s_{-i}\right)$ for some $s_{i} \in S_{i}$. (17)'s contraposition reads: $P(\tilde{s})<P(s) \Rightarrow$ $\tilde{s} \nsucceq$ s. Because $s_{-i}=\tilde{s}_{-i}$, the latter implies $\tilde{s}_{i} \notin \tilde{R}_{i}\left(s_{-i}\right)$. Another contradiction. We conclude that arg $\max _{s_{i} \in S_{i}} P\left(s_{i}, s_{-i}\right)=\tilde{R}_{i}\left(s_{-i}\right)$ for all $s_{-i} \in S_{-i}$.

\footnotetext{
21 The result appears to be new. Kukushkin (2004) defines a generalized best-reply potential game as a game which admits a function which satisfies (18). He also notes that this is a necessary condition for the game to be a best-reply potential game and sufficient for the game to be a pseudo-potential game (the latter can be seen by taking a single-valued selection in which case (17) becomes redundant).
} 
Let $\hat{S}_{i}$ denote the range of player $i$ 's best-reply map, i.e., the set $\left\{s_{i} \in S_{i}: s_{i} \in\right.$ $R_{i}\left(\sigma_{i}\left(s_{-i}\right)\right)$ for some $\left.s_{-i} \in S_{-i}\right\} \subseteq S_{i}{ }^{22}$ That $s_{i} \in \hat{S}_{i}$ means that $s_{i}$ is a best-reply to some vector of opponents' strategies. $\hat{S}_{i}$ is a compact set when $S_{-i}$ is compact and the best-reply correspondence is upper hemi-continuous (remember that $\sigma_{i}$ is continuous by definition).

As a first step in our proof, we will verify (17)-(18) under the additional requirement that when $\tilde{s} \succeq s(\tilde{s} \succ s), s_{i} \neq \tilde{s}_{i} \Rightarrow s_{i} \in \hat{S}_{i}$. Under this extra assumption, it is possible to argue along the lines of Kukushkin (2005) (see also Dubey et al. (2006), who adapt the potential function originally proposed in Huang (2002)). The potential function $\left(P^{R}\right.$ below) might be called a "restricted" best-reply potential (because it only works when $s_{i}$ is restricted as described).

When assumption $1^{\prime}$ holds, let $r_{i}, i \in \mathscr{I}$, denote the maximal selections from the $R_{i}$ 's (these maximal selections are decreasing under assumption $1^{\prime}$ ). If only the (weaker) assumption 1 holds, let instead $r_{i}, i \in \mathscr{I}$, denote the maximal selections from the sectioning of the closure of the graph of the decreasing selection $\hat{R}_{i}: X_{-i} \rightarrow$ $2^{S_{i}}$ (see footnote 13 for the details). Notice that the correspondence $\hat{R}_{i}$ is a subcorrespondence of $R_{i}$ (cf. lemma 1).

Let $\perp_{i}=\min _{s_{-i} \in S_{-i}} \sigma_{i}\left(s_{-i}\right), \top_{i}=\max _{s_{-i} \in S_{-i}} \sigma_{i}\left(s_{-i}\right)$, and extend each $r_{i}$ to $\left[\perp_{i}, \top_{i}\right]$ along the lines of Kukushkin (2005) (beginning of the proof of proposition 4.1). Under assumption 2, each function $F_{i}$ will be absolutely continuous in the restricted sense (see Gordon (1994)) in $x_{-i}$, and in particular $D_{1} F_{i}$ will exist almost everywhere. Taking $D_{1} F_{i}=0$ when it does not exist, the following restricted best-reply potential function is well-defined where the integral is the Henstock-Kurzweil integral (again see Gordon (1994) and also Jensen (2007a)). ${ }^{23}$

$$
P^{R}\left(s_{i}, s_{-i}\right)=\sum_{i}\left[\int_{\perp_{i}}^{\top_{i}} \min \left\{D_{1} F_{i}\left(\tau, s_{i}\right), D_{1} F_{i}\left(\tau, r_{i}(\tau)\right)\right\} d \tau+F_{i}\left(\perp_{i}, s_{i}\right)\right]-g\left(s_{i}, s_{-i}\right)
$$

Note that by assumption $2, D_{1} F_{i}\left(\tau, s_{i}\right) \leq D_{1} F_{i}\left(\tau, r_{i}(\tau)\right)$ if $s_{i} \leq r_{i}(\tau)$, and $D_{1} F_{i}\left(\tau, s_{i}\right) \geq$ $D_{1} F_{i}\left(\tau, r_{i}(\tau)\right)$ if $s_{i} \geq r_{i}(\tau)$. Take $s, \tilde{s} \in S$ with $\tilde{s} \succeq s, s_{i} \in \hat{S}_{i}$. If $s_{i}=\tilde{s}_{i}$ it is obvious that $P^{R}(s)=P^{R}(\tilde{s})$ so we are done. Let $\tilde{x}_{-i}=\sigma_{i}\left(s_{-i}\right)$, hence $\tilde{s}_{i} \in R_{i}\left(\tilde{x}_{-i}\right)$. Since $s_{i} \in \hat{S}_{i}$, there exists $x_{-i} \in X_{-i}$ such that $s_{i} \in R_{i}\left(x_{-i}\right)$. The fundamental theorem of calculus for the Henstock-Kurzweil integral (see again e.g. Gordon (1994) and Jensen (2007a)) applies, which allows us to conclude as follows:

$$
\begin{gathered}
P^{R}\left(\tilde{s}_{i}, s_{-i}\right)-P^{R}\left(s_{i}, s_{-i}\right)=F_{i}\left(\tilde{x}_{-i}, s_{i}\right)-F_{i}\left(\tilde{x}_{-i}, \tilde{s}_{i}\right)+ \\
\int_{\tilde{x}_{-i}}^{x_{-i}} D_{1} F_{i}\left(\tau, r_{i}(\tau)\right) d \tau+\int_{\perp_{i}}^{\tilde{x}_{-i}} D_{1} F_{i}\left(\tau, \tilde{s}_{i}\right) d \tau-\int_{\perp_{i}}^{x_{-i}} D_{1} F_{i}\left(\tau, s_{i}\right) d \tau+F_{i}\left(\perp_{i}, \tilde{s}_{i}\right)-F_{i}\left(\perp_{i}, s_{i}\right)= \\
\int_{\perp_{i}}^{\tilde{x}_{-i}} D_{1} F_{i}\left(\tau, s_{i}\right) d \tau+\int_{\tilde{x}_{-i}}^{x_{-i}} D_{1} F_{i}\left(\tau, r_{i}(\tau)\right) d \tau+\int_{\perp_{i}}^{\tilde{x}_{-i}} D_{1} F_{i}\left(\tau, \tilde{s}_{i}\right) d \tau-
\end{gathered}
$$

\footnotetext{
22 Clearly the range of $R_{i}$ coincides with the range of the actual best-reply map $\tilde{R}_{i}$.

23 If $F_{i}$ is $C^{1}$ we may use instead the Riemann integral. If it is absolutely continuous, the Lebesgue integral can be used. So the reader who is uncomfortable with the Henstock-Kurzweil integral can substitute the Riemann or Lebesgue integrals in the following.
} 


$$
\begin{gathered}
\int_{\perp_{i}}^{x_{-i}} D_{1} F_{i}\left(\tau, s_{i}\right) d \tau-\int_{\perp_{i}}^{\tilde{x}_{-i}} D_{1} F_{i}\left(\tau, \tilde{s}_{i}\right) d \tau= \\
\int_{\tilde{x}_{-i}}^{x_{-i}}\left[D_{1} F_{i}\left(\tau, r_{i}(\tau)\right)-D_{1} F_{i}\left(\tau, s_{i}\right)\right] d \tau
\end{gathered}
$$

It is clear that if $x_{-i}=\tilde{x}_{-i}$ (which happens if $s_{i}, \tilde{s}_{i} \in R_{i}\left(\sigma_{i}\left(s_{-i}\right)\right)$, i.e., if (17) holds but not (18)), then this term equals zero. If not, i.e., if $s_{i} \notin R_{i}\left(\sigma_{i}\left(s_{-i}\right)\right)$, there are two cases: If $\tilde{x}_{-i}>x_{-i}$ then $s_{i} \geq r_{i}(\tau)$ for all $\tau$ with strict inequality for all $\tau$ in an open neighborhood of $\tilde{x}_{-i}$. Since then $D_{1} F_{i}\left(\tau, r_{i}(\tau)\right) \leq D_{1} F_{i}\left(\tau, s_{i}\right)$ for a.e. $\tau \in\left[x_{-i}, \tilde{x}_{-i}\right]$, it is clear that (20) is greater than or equal to 0 . Assume to arrive at a contradiction that $(20)=0$. This will be the case if and only if $D_{1} F_{i}\left(\tau, r_{i}(\tau)\right)=D_{1} F_{i}\left(\tau, s_{i}\right)$ for a.e. $\tau \in\left[x_{-i}, \tilde{x}_{-i}\right]$. There exists a decreasing function $t:\left[x_{-i}, \tilde{x}_{-i}\right] \rightarrow \mathbb{R}_{+}$with $t\left(\tilde{x}_{-i}\right)=0$ and $t\left(x_{-i}\right)=s_{i}-\tilde{s}_{-i}>0$ such that $D_{1} F_{i}\left(\tau, r_{i}(\tau)\right)-D_{1} F_{i}\left(\tau, s_{i}\right) \geq D_{1} F_{i}\left(\tau, \tilde{s}_{i}+t(\tau)\right)-$ $D_{1} F_{i}\left(\tau, s_{i}\right)$ for all $\tau$. It follows that there is some $\alpha>0$ and some $\varepsilon>0$ such that for all $\tau \in\left[x_{-i}, x_{-i}+\varepsilon\right]: D_{1} F_{i}\left(\tau, r_{i}(\tau)\right)-D_{1} F_{i}\left(\tau, s_{i}\right) \geq D_{1} F_{i}\left(\tau, \tilde{s}_{i}+\alpha\right)-D_{1} F_{i}\left(\tau, s_{i}\right)$. Integrate to get $0 \geq F_{i}\left(x_{-i}+\varepsilon, \tilde{s}_{i}+\alpha\right)-F_{i}\left(x_{-i}+\varepsilon, s_{i}\right)-\left[F_{i}\left(x_{-i}, \tilde{s}_{i}+\alpha\right)-F_{i}\left(x_{-i}, s_{i}\right)\right]$. This contradicts assumption 2, so (20)>0.

If $\tilde{x}_{-i}<x_{-i}$ then $s_{i} \leq r_{i}(\tau)$ for all $\tau$ with strict inequality for all $\tau$ in an open neighborhood of $x_{-i}$. The proof is similar in this case and is omitted.

We conclude that when $\left(\tilde{s}_{i}, s_{-i}\right),\left(s_{i}, s_{-i}\right) \in S$ and $s_{i} \in \hat{S}_{i},\left(\tilde{s}_{i}, s_{-i}\right) \succeq(\succ)\left(s_{i}, s_{-i}\right) \Rightarrow$ $P^{R}\left(\tilde{s}_{i}, s_{-i}\right)-P^{R}\left(s_{i}, s_{-i}\right) \geq(>) 0$. So the conditions of lemma 1 have been verified for the "restricted" case.

Remark 61 There is one case when the potential $P^{R}$ applies directly (and in particular, the potential will then be a continuous function since so is $\left.P^{R}\right)$. This is when the selection $r_{i}$ of assumption 1 is continuous. Such a selection will map onto an interval which allows one to extend the domain of the reduced best-reply selections and take $\hat{S}_{i}=S_{i}$ for all $i$ above (see Dubey et al. (2006), p.82, l.6-8 for details. This clearly works whether the aggregator is linear or not).

Next we define a potential function which applies to the general case. Since $P^{R}$ is a continuous function, $v=\max _{s \in S} P^{R}(s)-\min _{s \in S} P^{R}(s)+1$ is a well-defined quantity (strictly greater than 1$)$. Now define:

$$
P(s)=P^{R}(s)+\sum_{i} \chi_{i}\left(s_{i}\right)
$$

where $\chi_{i}\left(s_{i}\right)=v$ if $s_{i} \in \hat{S}_{i}$ and $\chi_{i}\left(s_{i}\right)=0$ if $s_{i} \notin \hat{S}_{i}$. It is easy to see that $P$ will satisfy (17) and (18) for all $\tilde{s}, s \in S$. So $P$ is a best-reply potential. Furthermore, $P$ is upper semi-continuous because it is the sum of a continuous function $P^{R}$ and an upper semicontinuous function $\sum_{i} \chi_{i}\left(s_{i}\right)$. That $\sum_{i} \chi_{i}\left(s_{i}\right)$ is upper semi-continuity can be verified directly from the definition: The sets $\left\{s \in S: \sum_{i} \chi_{i}\left(s_{i}\right) \geq \alpha\right\}$ will be a finite union of sets of the type $\hat{S}_{A} \times S_{-A}, A \subseteq\{1, \ldots, I\}$ all of which are closed (since so are $\hat{S}_{i}$ and $S_{i}$ for all $i$ ). But then $\left\{s \in S: \sum_{i} \chi_{i}\left(s_{i}\right) \geq \alpha\right\}$ will be closed for any $\alpha \in \mathbb{R}$. 


\subsection{Proof of Theorem 2}

The following is an adaption of a standard argument from Liapunov methods (see e.g., Agerwal (2000)).

Lemma 2 Let $\mathbf{s}$ be any sequential improvement path (including weak ones and indifference paths). Then $\Omega(\mathbf{s}) \subseteq L(p) \equiv\{s \in S: P(s)=p\}$, some $p<+\infty$.

Proof: Let $\left(s^{t_{l}}\right)$ be a convergent subsequence of $S$ with limit point $s^{\omega} .\left(P\left(s^{t}\right)\right)_{t=0}^{\infty}$ is a non-decreasing sequence by (17), hence so is $\left(P\left(s^{t_{l}}\right)\right)_{l=0}^{\infty}$ and it follows that $\lim _{l \rightarrow \infty} P\left(s^{t_{l}}\right)=\lim _{t \rightarrow \infty} P\left(s^{t}\right)=p$. It is a direct consequence of the continuity of $P^{R}$ that $P\left(s^{t_{l}}\right) \rightarrow P(s)$ for any convergent subsequence $s^{t_{l}} \rightarrow s$ of a sequential improvement path $\left(s^{t}\right)_{t=0}^{\infty}$. In particular, $P\left(s^{\omega}\right)=p$ where by compactness, $p<+\infty$.

We are now ready to prove the theorem. Let $\mathbf{s}=\left(s^{t}\right)_{t=0}^{\infty}$ be an admissible sequential improvement path and $\left(s^{t_{l}}\right)$ a convergent subsequence with limit $s$. Clearly $s^{t_{l}+1} \in\left(R_{i\left(t_{l}\right)}\left(\sigma_{i\left(t_{l}\right)}\left(s_{-i\left(t_{l}\right)}^{t_{l}}\right)\right),\left\{s_{-i\left(t_{l}\right)}^{t_{l}}\right\}\right)$ for all $l$. Since the sequence is infinite and the number of players is finite, we may pick a subsequence $\left(s^{t_{m}}\right)_{m \in \mathbb{N}}$ such that $i\left(t_{l_{m}}\right)=1$, say, for all $m$. It follows that $s^{t_{m}+1} \in\left(R_{1}\left(\sigma_{1}\left(s_{-1}^{t_{l}}\right)\right),\left\{s_{-1}^{t_{l m}}\right\}\right)$ for all $m$. Since the best-reply correspondences are upper hemi-continuous, it is true that $s^{t_{m}+1} \rightarrow$ $\tilde{s}^{1} \in\left(R_{1}\left(\sigma_{1}\left(s_{-1}\right)\right),\left\{s_{-1}\right\}\right)$ as $m \rightarrow \infty$ (for any subsequence such that the limit is well-defined. Such a subsequence is chosen and indexed here again by $\left.l_{m}\right)$. Note that $\{s, \tilde{s}\} \subseteq \Omega(\mathbf{s})$, so by lemma $2, P(s)=P(\tilde{s})$. But then, because of (17)-(18), it must be the case that $s_{1}, \tilde{s}_{1} \in R_{1}\left(\sigma_{1}\left(s_{-1}\right)\right)$. At every date $t_{l_{m}}$, player 1 moves by construction; and passing to yet another subsequence if necessary, we can therefore find another player, say $i=2$, who moves at all $t_{l_{1_{m}}}+1$ (admissibility). Now we repeat the previous argument, beginning with the observation that $\left(s^{t_{l m}}{ }^{+1}\right) \rightarrow \tilde{s}^{1}$ is itself a convergent subsequence of $\mathbf{s}$ (in particular, $\tilde{s}^{1} \in \Omega(\mathbf{s})$ ). This yields a limit $\tilde{s}^{2}$ with the property that $s_{2}, \tilde{s}_{2}^{2} \in R_{2}\left(\sigma_{2}\left(\tilde{s}_{-2}^{1}\right)\right)$. Again $\tilde{s}^{2} \in \Omega(\mathbf{s})$ (being the limit of a convergent subsequence). This argument can be continued indefinitely, yielding an admissible indifference path $\left(\tilde{s}^{t}\right)_{t=1}^{\infty}$ fully contained in $L(p)$. Under the assumptions of the theorem, any indifference path is trivial (the infinite repetition of a PSNE). This leaves only one possibility, namely that $s^{0}$ is a PSNE.

\section{References}

Aczél, J., Lectures on Functional Equations and Their Applications, Dover Publications, Mineola, 2006. (Unabridged version of the original 1966 Academic Press Edition)

Agerwal, R.P., Difference Equations and Inequalities: Theory, Methods, and Applications, 2nd Edition, Marcel Dekker: New York, 2000.

Alos-Ferrer, C. and A.B. Ania (2005): "The Evolutionary Stability of Perfectly Competitive Behaviour", Economic Theory, 26, 497-516.

Amir, R. (1996): "Sensitivity Analysis of Multisector Optimal Economic Dynamics", Journal of Mathematical Economics 25, 123-141. 
Cornes, R. and R. Hartley (2001): "Disguised Aggregative Games", Discussion Paper 1/11, Univ. of Nottingham.

Cournot, A., Recherches sur les Principes Mathematiques de la Theorie des Richesses, Paris: Hachette, 1838. (English translation by N. T. Bacon, Macmillan, 1897).

Dubey, P., O. Haimanko, and A. Zapechelnyuk (2006): "Strategic Complements and Substitutes, and Potential games", Games and Economic Behavior 54, 77-94.

Edlin, A. and C. Shannon (1998) : "Strict Monotonicity in Comparative Statics", Journal of Economic Theory 81, 201-219.

Gordon, R.A., The integrals of Lebesgue, Denjoy, Perron, and Henstock, American Mathematical Society, Providence, 1994.

Gorman, W.M. (1968): "The Structure of Utility Functions", Review of Economic Studies 35, 367-390.

Huang, Z., Fictitious play in games with a continuum of strategies, Ph.D. Thesis, Dep. of Economics, State University of New York at Stony Brook, 2002.

Jensen, M.K. (2006): “Aggregative Games", Discussion Paper No. 06-10, University of Birmingham.

Jensen, M.K. (2007a): "Monotone Comparative Statics in Ordered Vector Spaces", The B.E. Journal of Theoretical Economics 7:1, Article 35, 2007.

Jensen, M.K. (2007b): "Stability of Pure Strategy Nash Equilibrium in Best-reply Potential Games", Mimeo, University of Birmingham.

Kockesen, L., E. Ok, and R. Sethi (2000): "Evolution of Interdependent Preferences in Aggregative Games", Games and Economic Behavior 31, 303-310.

Kukushkin, N.S. (1994): "A Fixed-point Theorem for Decreasing Mappings", Economics Letters 46, 23-26.

Kukushkin, N.S. (2004): "Best Response Dynamics in Finite Games with Additive Aggregation", Games and Economic Behavior 48, 94-110.

Kukushkin, N.S. (2005): "'Strategic supplements" in games with polylinear interactions", Mimeo, Russian Academy of Sciences.

Morris, S. and T. Ui (2004): "Best response equivalence", Games and Economic Behavior 49, 260-287.

Novshek, W. (1985): "On the Existence of Cournot Equilibrium", Review of Economic Studies 52, 86-98.

Possajennikov, A. (2003): "Evolutionary Foundation of Aggregative-taking Behavior", Economic Theory 21, 921-928.

Schipper, B. (2005): "The Evolutionary Stability of Optimism, Pessimism and Complete Ignorance", Mimeo, University of California at Davis.

Topkis, D.M., Supermodularity and Complementarity, Princeton University Press, New Jersey, 1998.

Vind, K. and B. Grodal, Independence, Additivity, Uncertainty, Springer Verlag (Studies in Economic Theory Vol.14), Berlin, 2003.

Voorneveld, M. (2000): "Best-response Potential Games", Economics Letters 66, 289-295. 